ISSN: $2394-2258$

Available at http://scientificadvances.co.in

DOI: http://dx.doi.org/10.18642/ijamml_7100121740

\title{
AN IMPROVED SIMULATION MODEL OF RAYLEIGH FADING CHANNELS
}

\section{Cuijuan Guo, Zhen Yang and Zhigang Wu}

School of Electronics and Information Engineering, Tianjin Polytechnic University, P. R. China

\begin{abstract}
The model of propagation of electromagnetic energy from transmitter to receiver will be largely by way of scatting, either by reflection from the flat sides of buildings or by diffraction around such buildings or other man-made or natural obstacles in mobile communication. These effects are called multi path propagation resulting in significant fluctuations of the received signal. Generally, Rayleigh fading waveforms are often required to simulate in wireless communication channels. In this paper, in order to tackle with statistical property problems of the proposed model that cannot always approach theoretical value, an improved model based on Clarke model is proposed and its statistical properties of first and second-order are also analyzed in detail. Simulation results indicate that the modified amplitude, phase probability density function, the relevant function, the level crossing rate and the average fade duration can coincide with Clarke theoretical model characteristics. It can meet the requirements of the actual channel simulation.
\end{abstract}

Keywords: mobile communication, Rayleigh fading, Clarke model, statistical properties.

\footnotetext{
${ }^{*}$ Corresponding author.

E-mail address: 2256570959@qq.com (Cuijuan Guo).
}

Copyright () 2016 Scientific Advances Publishers

2010 Mathematics Subject Classification: 93C62, 94A12, 94A40.

Submitted by Loc Nguyen.

Received November 18, 2016 


\section{Introduction}

Wireless communications have become one of the most indispensable technologies in our modern life. Many new systems, such as automated highways and factories, wireless sensor networks, remote telemedicine, and smart building and appliances, are emerging from ideas of wireless communications research. In order to improve the quality of communications systems, network researchers and designers need to evaluate the radio channel performance. Generally, statistical model of mobile-radio channels is used for stimulating wireless communication systems and is of importance to the software and hardware realization [1]-[3]. The main cause is that the transmitted signal is not a single path of the reflected wave to reach the mobile station antenna, but the synthesis of many paths, due to the fact that the wireless channels depend on radio propagation models, such as reflections from a smooth surface, line of sight radiation, scattering caused by objects, and diffractions around a corner. Its propagation model can be divided into two categories: large-scale fading and small-scale fading. The former describes the slow changes in the intensity of the received signal within a long distance (several hundred meters). In general, large-scale fading is inversely proportional to distance between the transmitting antennas and the receiving antennas, and has different attenuation factor in different regions. The latter indicates the rapid fluctuations of the received signal strength within a short distance (several wavelength) or short time (in seconds). According to the channel frequency selectivity, the small-scale fading channel can be divided into flat fading channel and frequency selective fading channel. The simulation model of frequency selective fading can be composed of several flat fading models with different time delays. Based on this, we mainly study the flat fading channel model. The envelope curve of the channel response is subject to Rayleigh fading, and the phase is uniformly distributed on $[\pi \pi]$. From the above, we can know the key to radio channel model is how to efficiently generate the Rayleigh fading envelope. Clarke proposed a statistical model for the description of flat small-scale fading when $N$ approaches infinity, making it impossible to use hardware and software 
implementation [4], but it can be used as a basis for a variety of simulation models. Subsequently Jakes' method and its derivatives are designed for deterministic sum-of-sinusoids (SoS) channel simulators [5][8], which have the advantage of simulation efficiency. However, these channel simulators still retain some undesirable properties. For example, the cross-correlation function (CCF) of any pair of underlying complex processes is generally not zero and the in-phase and quadrature components of each underlying complex process have different autocorrelation functions (ACF). To remedy the shortcomings of the deterministic channel simulators in [5]-[8], non-ergodic stochastic SoS channel simulators is proposed in [9]. By averaging over a large number of simulation trials, the developed stochastic channel simulators can approximate the desired statistical properties. However, relatively high computational complexity has to be paid for the channel simulators due to its non-ergodic stochastic nature [10]. Additionally, Pop and Beaulieu put forward to add random phase on each low frequency oscillator and eliminate the problem of stability in [11]. Based on this, the literature [12] has proved that the second-order statistical properties of the model are not consistent with the theoretical model.

In this paper, an improved simulation is proposed for producing multiple uncorrelated Rayleigh fading waveforms without using large numbers of sinusoids for SoS channel simulators. The simulation results highlight the advantages of the improved channel simulator over other forms of channel simulators in both accurate reproduction of all the desired statistical properties of the reference model and efficient implementation.

\section{Clarke Reference Model}

It is well-known that Rayleigh fading channel models, characterized by the complex Gaussian progress $g(t)$, can be modelled and efficiently simulated by using the SoS process

$$
g(t)=\sum_{n=1}^{N} C_{n} e^{\left[j\left(\omega_{d} t \cos \alpha_{n}+\varphi_{n}\right)\right]}
$$


where $N$ is defined the number of sinusoids, $C_{n}$ is called the gain, $\omega_{d}=2 \pi f_{m}, f_{m}$ is referred to as the maximum Doppler frequency that is related to the vehicle speed $v$ by $f_{m}=\frac{v}{\lambda}$, where $\lambda$ is the carrier wave length. $\alpha_{n}$ indicates the arrival angle of the incoming waves and the phases $\varphi_{n}$ is independent and identical distribution over $[-\pi, \pi)$.

In (1), the phase component and quadrature component are given by

$$
\begin{aligned}
g_{c}(t) & =\sum_{n=1}^{N} C_{n} \cos \left(\omega_{d} t \cos \alpha_{n}+\varphi_{n}\right), \\
g_{s}(t) & =\sum_{n=1}^{N} C_{n} \sin \left(\omega_{d} t \cos \alpha_{n}+\varphi_{n}\right) .
\end{aligned}
$$

Provided that $N$ is sufficiently large, the central limit theorem justifies that the components of $g(t)$ can be approximated as Gaussian random processes, whose mean-value is zero and variance is one. Furthermore, the $C_{n}$ is normalized so that the ensemble average $\sum_{n=0}^{N} E\left[C_{n}^{2}\right]=1$. Complex envelop $|g(t)|$ of Rayleigh distribution can be written as

$$
f_{|g(t)|}(t)=r \exp \left(-\frac{r^{2}}{2}\right), r \geq 0
$$

It is reasonable that the waves can arrive from any direction with equal probability. Therefore, the probability density function (PDF) of the fading phase, $\psi(t)=\tan ^{-1}\left[g_{c}(t), g_{s}(t)\right]$, is uniformly distributed over $[-\pi, \pi)$.

Equation (1) has the following second-order statistical properties [13]:

$$
\begin{aligned}
& R_{g_{c} g_{c}}(\tau)=J_{0}\left(\omega_{d} \tau\right), \\
& R_{g_{s} g_{s}}(\tau)=J_{0}\left(\omega_{d} \tau\right), \\
& R_{g_{c} g_{s}}(\tau)=R_{g_{s} g_{c}}(\tau)=0,
\end{aligned}
$$




$$
\begin{aligned}
& R_{g g}(\tau)=2 J_{0}\left(\omega_{d} \tau\right), \\
& R_{|g|^{2}|g|^{2}}(\tau)=4+4 J_{0}^{2}\left(\omega_{d} \tau\right),
\end{aligned}
$$

where $J_{0}(\cdot)$ denotes the Bessel function of the first kind and zero-order. The first and second-order statistical properties of the reference model are discussed above, and an efficient simulation of the Rayleigh fading channel is needed to satisfy the above statistics properties.

\section{Proposed Model}

For simplicity, it will be assumed that at every points there are exactly $N$ component waves and these $N$ waves have the same amplitude. In addition, we assume that the transmitted radiation is vertically polarized. Likewise, the total field at any receiving points is also vertically polarized and is composed of the superposition of $N$ waves. This paper shows that the scattering model can be used to predict the statistical characteristics of the received signal at the antenna terminals. These characteristics include the probability distributions of amplitude and phase, correlation functions, the level cross rate and the average fade duration. In this section, an improved simulation model is proposed by reintroducing the randomness compared with Zheng's model [14]. As we know, in cellular land mobile-radio systems, the radio signals will propagate in three dimensions. However, if the distance between the base station (BS) and mobile station (MS) is sufficiently large, the radio propagation environment is often modelled as occurring in a $2 \mathrm{D}$ plane. Furthermore, we assume that there are also $N$ independent fading waveforms, where $N=4 M, M$ is the number of sinusoids in one quadrant. We adjust initial phase as follows: $\varphi_{n}=\phi_{n}+\frac{\pi}{2}, \varphi_{n+M}=-\varphi_{n}, \varphi_{n+2 M}=-\phi_{n}+\frac{\pi}{2}$, $\varphi_{n+3 M}=\varphi_{n}$, and we also define the initial arrival phase as $\alpha_{n}=\frac{2 \pi n+\theta}{N}$, where $\theta$ is uniformly distributed on $[-\pi, \pi)$. Therefore, Equation (1) can be also written as shown: 


$$
\begin{aligned}
Z(t)= & \sqrt{\frac{2}{N}}\left\{\sum_{n=1}^{M} e^{\left[j\left(\omega_{d} t \cos \alpha_{n}+\varphi_{n}\right)\right]}+\sum_{n=M-1}^{2 M} e^{\left[j\left(\omega_{d} t \cos \alpha_{n}+\varphi_{n}\right)\right]}\right. \\
& \left.+\sum_{n=2 M-1}^{3 M} e^{\left[j\left(\omega_{d} t \cos \alpha_{n}+\varphi_{n}\right)\right]}+\sum_{n=3 M-1}^{4 M} e^{\left[j\left(\omega_{d} t \cos \alpha_{n}+\varphi_{n}\right)\right]}\right\} \\
= & \sqrt{\frac{2}{N}}\left\{\sum_{n=1}^{M} e^{\left[j\left(\omega_{d} t \cos \alpha_{n}+\phi_{n}+\frac{\pi}{2}\right)\right]}+\sum_{n=1}^{M} e^{\left[-j\left(\omega_{d} t \sin \alpha_{n}+\varphi_{n}\right)\right]}\right. \\
& \left.+\sum_{n=1}^{M} e^{\left[-j\left(\omega_{d} t \cos \alpha_{n}+\phi_{n}-\frac{\pi}{2}\right)\right]}+\sum_{n=1}^{M} e^{\left[j\left(\omega_{d} t \sin \alpha_{n}+\varphi_{n}\right)\right]}\right\} \\
= & \sqrt{\frac{2}{M}}\left\{\sum_{n=1}^{M} \cos \left(\omega_{d} t \sin \alpha_{n}+\varphi_{n}\right)+j \cdot \sum_{n=1}^{M} \cos \left(\omega_{d} t \cos \alpha_{n+\phi_{n}}\right)\right\} .
\end{aligned}
$$

The in-phase and quadrature components can be expressed as

$$
\begin{aligned}
& Z_{c}(t)=\sqrt{\frac{2}{M}} \sum_{n=1}^{M} \cos \left(\omega_{d} t \sin \alpha_{n}+\varphi_{n}\right), \\
& Z_{s}(t)=\sqrt{\frac{2}{M}} \sum_{n=1}^{M} \cos \left(\omega_{d} t \cos \alpha_{n}+\phi_{n}\right),
\end{aligned}
$$

where $\alpha_{n}=\frac{2 \pi n+\theta}{N}, n=1,2, \ldots, M ; \varphi_{n}, \phi_{n}$, and $\theta$ are mutually independent and uniformly distributed over $[-\pi, \pi)$.

We have known that it is necessary to design a complex Gaussian random process to simulate flat fading channel. At any moment $t, Z_{c}(t)$ and $Z_{s}(t)$ are composed of $M$ random variables, each of them is independent and has the same mean and variance. According to the central limit theorem, $Z_{c}(t)$ and $Z_{s}(t)$ are Gauss random variables when $M$ approaches infinity. The one-dimensional distribution of complex envelope $Z(t)$ is Rayleigh distribution. It can be expressed as 


$$
f_{Z}(r)=r \cdot \exp \left(-\frac{r^{2}}{2}\right)
$$

And the one-dimensional phase distribution is uniform distribution. It can be written as

$$
f_{Z}(\xi)=\frac{1}{2 \pi}
$$

The detailed calculation processes of the correlation function are in Appendix A.

From above calculation results, we can know even when $M$ is a finite value, $R_{Z_{c} Z_{c}}(\tau), R_{Z_{s} Z_{s}}(\tau), R_{Z_{c} Z_{s}}(\tau)$, and $R_{Z Z}(\tau)$ are also fully consistent with the values of the reference model. $R_{|z|^{2}|z|^{2}}(\tau)$ is also equal to the reference value when $M$ tends to infinity.

The probability density functions of amplitude and phase indicate the characteristics of fading channel at different time. While the level cross rate (LCR) and the average fade duration (AFD) represent the changing level speed, which are two important second-order statistical properties.

The LCR, denoted here by $N_{R}(r)$, provides us with a measure of the average number of crossings per second at which the envelope crosses a specified signal level $r$ with positive slope. It is given by

$$
N_{R}(r)=\int_{0}^{\infty} \dot{r} p(R, \dot{r}) d_{\dot{r}}=\sqrt{2 \pi f_{\max }} \rho e^{-\rho^{2}},
$$

where the dot indicates the time derivative and $p(R, \dot{r})$ is the joint density function of $r$ and $\dot{r}$ at $r=R$. $\rho$ is the ratio root mean square for level $R$ and amplitude fading.

The AFD, denoted here by $T_{R}(r)$, is the mean value for the length of all time intervals over which the signal envelop is below a specified level $R$. Then the probability is

$$
P_{r}(r \leq R)=\int_{0}^{R} P(r) d_{r}=1-e^{-\rho^{2}} .
$$


The average fade duration is

$$
T_{R}=\frac{P_{r}(r \leq R)}{N_{R}(r)} .
$$

Substituting (12) and (13) for (14), we get

$$
T_{R}(r)=\frac{e^{\rho^{2}}-1}{\sqrt{2 \pi} \rho f_{\max }} .
$$

\section{Performance Evaluation}

To evaluate the performance of the proposed fading model, simulations are carried out to generate 8 fading waveforms with the following parameters: $M=8, f_{m}=15 \mathrm{~Hz}$ (corresponding to a carrier frequency of $900 \mathrm{MHz}$ and a vehicle speed of $60 \mathrm{~km} / \mathrm{h}$ ). And 5000 samples are generated for each waveform with the sampling rate $9.6 \mathrm{KHz}$. In order to distinguish the models, the improved model and Zheng's model are defined as Model I and Model II, respectively.

In Figure 1, it can be seen envelope's PDFs of proposed model and Zheng's model are nearly to the Rayleigh distribution. The probability density functions of the fading waveform's phase are also tested and the result is shown in Figure 2, which shows that the phase probability density function of the proposed model is very close to the theoretical value compared with Zheng's model. 


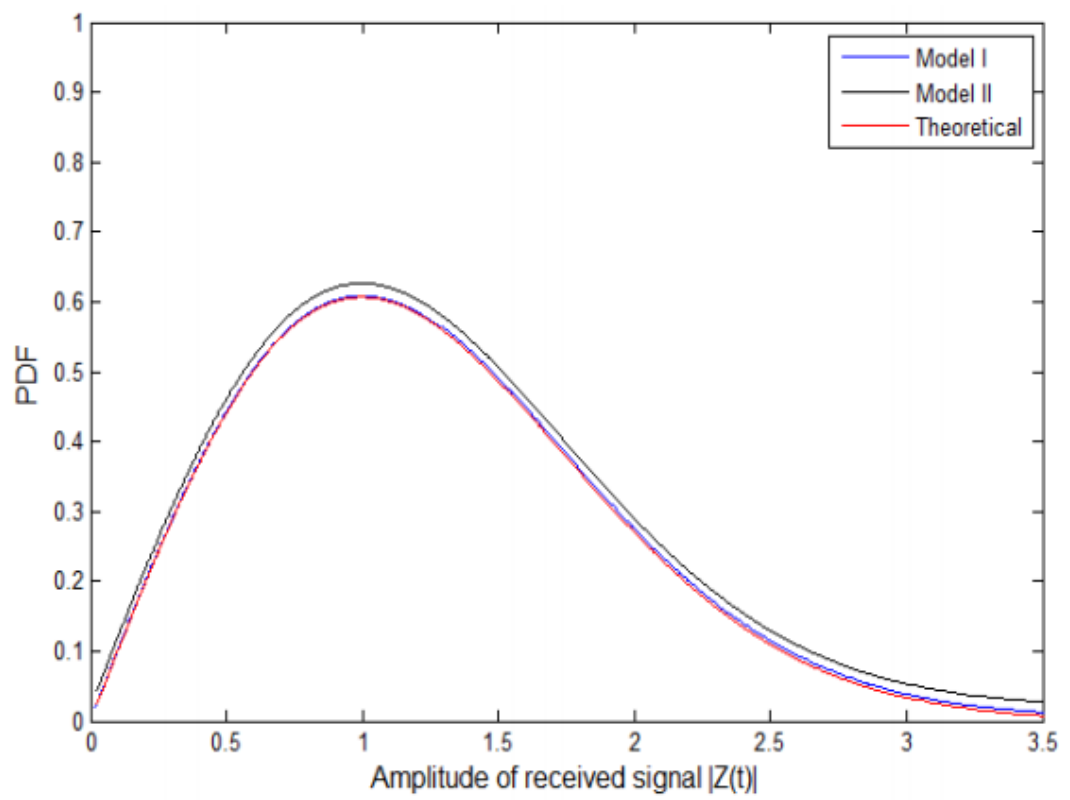

Figure 1. PDF of the amplitude of the fading envelope.

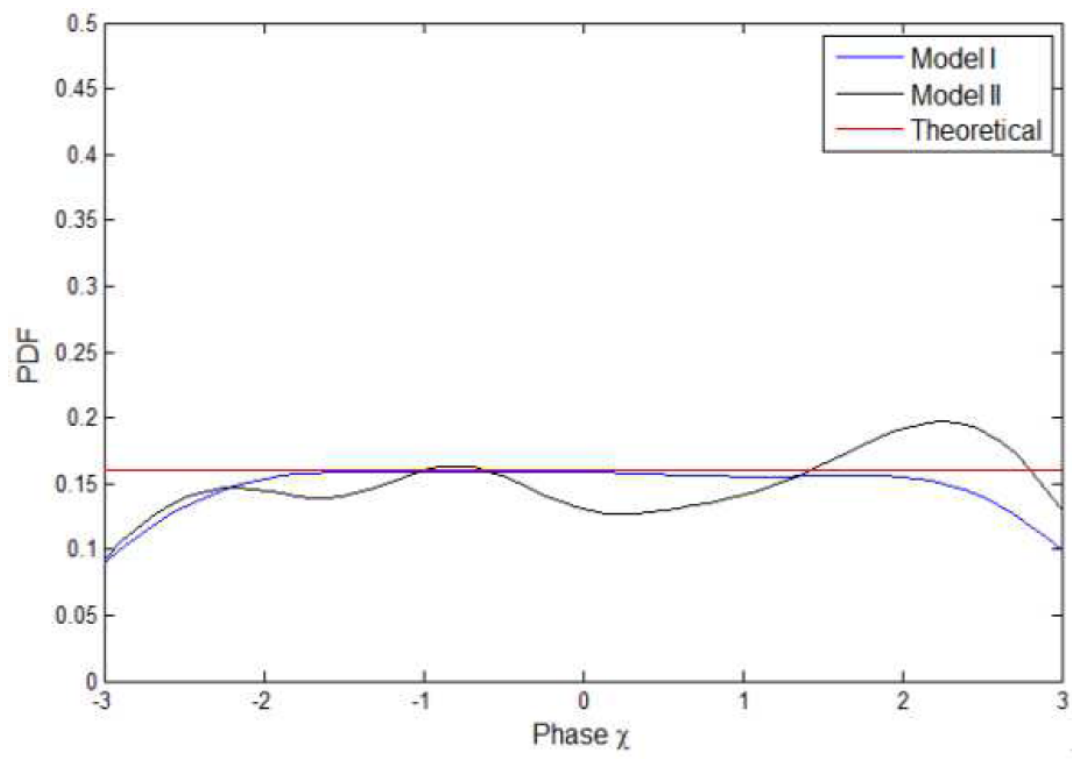

Figure 2. PDF of the phase. 
We now investigate the performance of the different models in terms of their autocorrelation functions. From Figure 3 and Figure 4, even though the number of sinusoids and the number of random trials are small, we can see that the autocorrelation functions of the in-phase and quadrature components of Model I are almost same as the theoretical Bessel function $J_{0}\left(\omega_{d} \tau\right)$. Clearly, we can conclude that Model I performs better than Model II in spite of their same relative operational complexity.

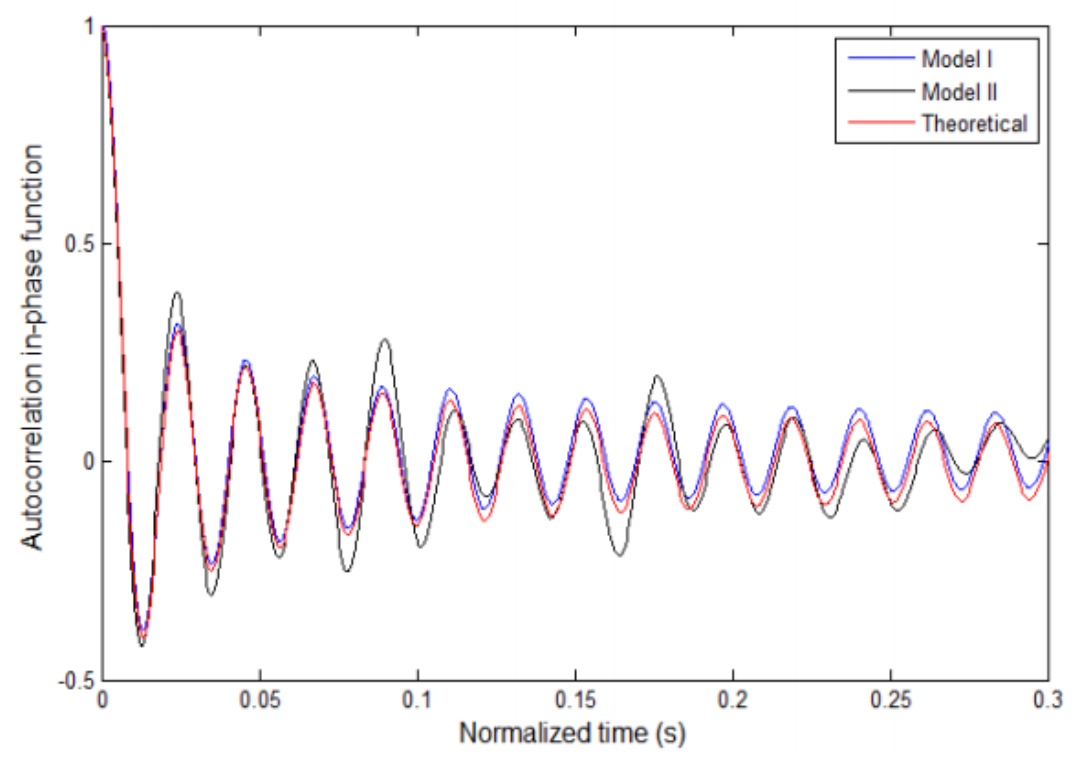

Figure 3. Comparison of autocorrelation function of real part. 


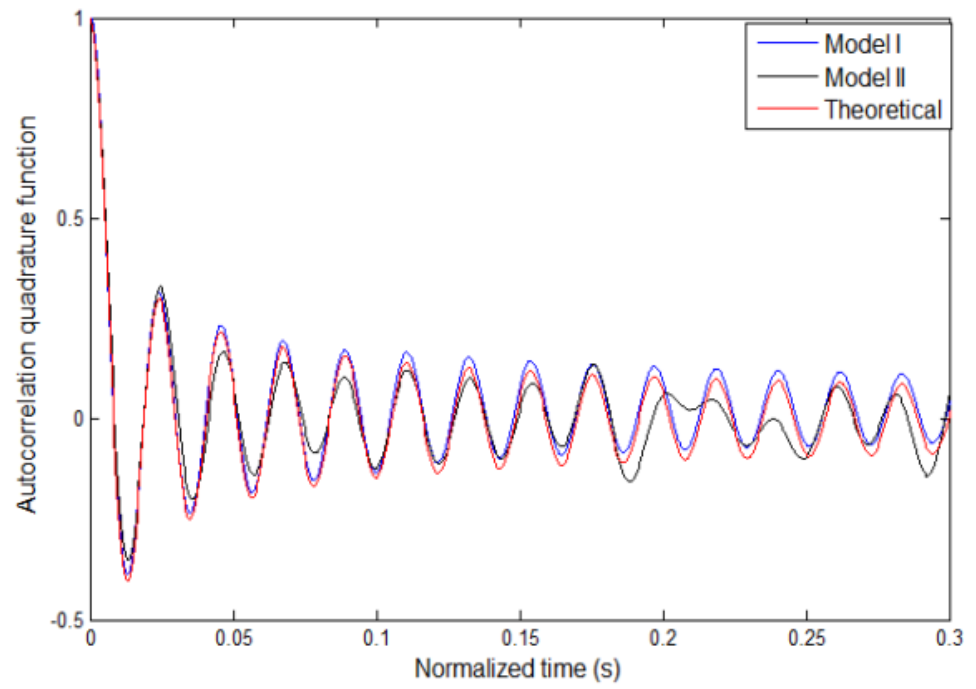

Figure 4. Comparison of autocorrelation function of image part.

Two important statistical properties of the simulated fading waveforms are also evaluated. Compared with theoretical values and Zheng's, it can be verified that the curve of the improved model fit the reference curve better than the Zheng' from Figure 5 and Figure 6.

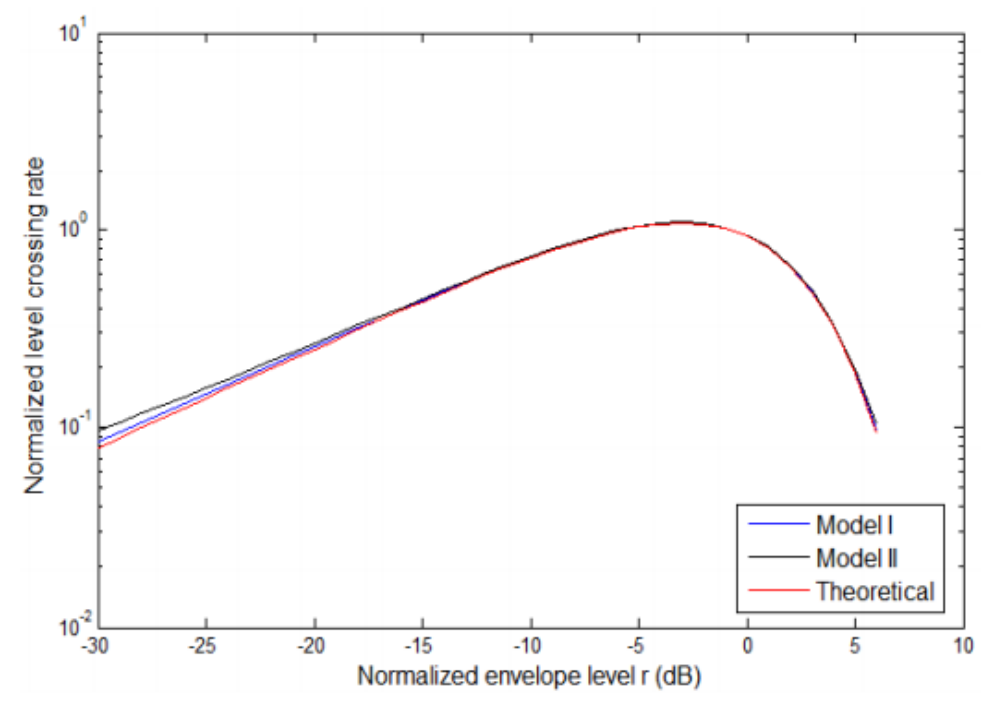

Figure 5. LCR of the fading envelope. 


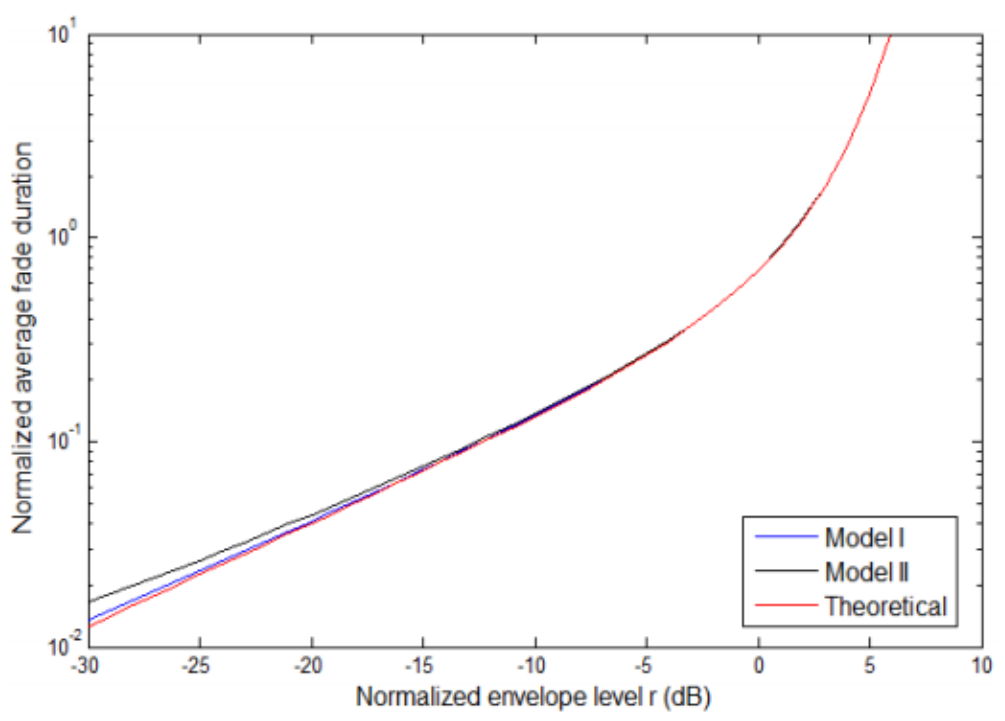

Figure 6. AFD of the fading envelope.

\section{Conclusion}

In this paper, an improved sum-of-sinusoid based on channel simulators has been presented, which is available in the literature for simulating flat-fading Rayleigh fading mobile-radio channels. By use of arrival angle arrangement and appropriately chosen incident wave phases, the in-phase and quadrature components in any fader are dependent and they also have almost identical functions. Higher order statistical properties that are superior to Zheng's including LCR and AFD have been analyzed, which can be helpful for coding scheme and interleaving depth. Furthermore, such a channel simulator that can be extended to frequency-selective channels would play an important role in the design of measurement based channel simulators. Hence, the channel simulator can be applied to a wide range of different propagation environments. 


\section{Appendix A: Derivation of Correlation Function}

The autocorrelation function of the in-phase component is as follows:

$$
\begin{aligned}
R_{Z_{c} Z_{c}}(\tau) & =E\left[Z_{c}(t) \cdot Z_{c}(t+\tau)\right] \\
& =\frac{2}{M} \sum_{n=1}^{M} \sum_{i=1}^{M} E\left[\cos \left(\omega_{d} t \sin \alpha_{n}+\varphi_{n}\right) \cdot \cos \left(\omega_{d}(t+\tau) \sin \alpha_{i}+\varphi_{i}\right)\right] \\
& =\frac{2}{M} \sum_{n=1}^{M} E\left[\cos \left(\omega_{d} t \sin \alpha_{n}+\varphi_{n}\right) \cdot \cos \left(\omega_{d}(t+\tau) \sin \alpha_{n}+\varphi_{n}\right)\right] \\
& =\frac{1}{M} \sum_{n=1}^{M} E\left[\cos \left(\omega_{d} \tau \sin \alpha_{n}\right)\right]=J_{0}\left(\omega_{d} \tau\right)
\end{aligned}
$$

Similarly, we can get the autocorrelation function of the quadrature component

$$
R_{Z_{s} Z_{s}}(\tau)=J_{0}\left(\omega_{d} \tau\right)
$$

As we all know, the in-phase and quadrature components in any fader of effective simulation models should be independent. That is to say, the computational result of cross-correlation function between inphase and quadrature components should be zero. Now, we adopt similar operations to derivate the calculation process as follows:

$$
\begin{aligned}
R_{Z_{c} Z_{s}}(\tau)= & E\left[Z_{c}(t) \cdot Z_{s}(t+\tau)\right] \\
= & \frac{2}{M} \sum_{n=1}^{M} \sum_{i=1}^{M} E\left[\cos \left(\omega_{d} t \sin \alpha_{n}+\varphi_{n}\right) \cdot \cos \left(\omega_{d}(t+\tau) \cos \alpha_{i}+\varphi_{i}\right)\right] \\
= & \frac{2}{M} \sum_{n=1}^{M} E\left\{\operatorname { c o s } ( \omega _ { d } t \operatorname { s i n } \alpha _ { n } + \varphi _ { n } ) \cdot \left[\cos \left(\omega_{d} t \cos \alpha_{n}+\varphi_{n}\right)\right.\right. \\
& \left.\left.\cdot \cos \left(\omega_{d} \tau \cos \alpha_{n}\right)-\sin \left(\omega_{d} t \cos \alpha_{n}+\varphi_{n}\right) \cdot \sin \left(\omega_{d} \tau \cos \alpha_{n}\right)\right]\right\}=0
\end{aligned}
$$


After similar calculation, we can also get

$$
R_{Z_{s} Z_{c}}(\tau)=0
$$

The complex envelope autocorrelation function of the improved model is as follows:

$$
\begin{aligned}
R_{Z Z}(\tau)= & E[Z(t) \cdot Z(t+\tau)]=E\left\{\left[Z_{c}(t)+j Z_{s}(t)\right] \cdot\left[Z_{c}(t+\tau)+j Z_{s}(t+\tau)\right]\right\} \\
= & E\left[Z_{c}(t) Z_{c}(t+\tau)\right]+E\left[Z_{s}(t) Z_{s}(t+\tau)\right]+j E\left[Z_{s}(t) Z_{c}(t+\tau)\right] \\
& -j E\left[Z_{c}(t) Z_{s}(t+\tau)\right]=2 J_{0}\left(\omega_{d} \tau\right) .
\end{aligned}
$$

The autocorrelation of the squared envelope is

$$
\begin{aligned}
R_{|z|^{2}|z|^{2}}(\tau)=E\left[Z_{c}^{2}(t) Z_{c}^{2}(t+\tau)\right]+E\left[Z_{s}^{2}(t) Z_{s}^{2}(t+\tau)\right] & +E\left[Z_{c}^{2}(t) Z_{s}^{2}(t+\tau)\right] \\
& +E\left[Z_{s}^{2}(t) Z_{c}^{2}(t+\tau)\right]
\end{aligned}
$$

where

$$
\begin{aligned}
E\left[Z_{c}^{2}(t) Z_{c}^{2}(t+\tau)\right]= & \frac{4}{M^{2}} E\left\{\sum_{n=1}^{M} \cos \left(\omega_{d} t \sin \alpha_{n}+\varphi_{n}\right) \cdot \sum_{i=1}^{M} \cos \left(\omega_{d} t \sin \alpha_{i}+\varphi_{i}\right)\right. \\
& \left.\cdot \sum_{p=1}^{M} \cos \left(\omega_{d} t \sin \alpha_{p}+\varphi_{p}\right) \cdot \sum_{q=1}^{M} \cos \left(\omega_{d} t \sin \alpha_{q}+\varphi_{q}\right)\right\} \cdot(21)
\end{aligned}
$$

It can be divided into four kinds of situations as follows:

$$
\begin{aligned}
& \text { (1) } n=i=p=q \\
& E\left[Z_{c}^{2}(t) Z_{c}^{2}(t+\tau)\right] \\
= & \frac{4}{M^{2}} \sum_{n=1}^{M} E\left\{\cos ^{2}\left(\omega_{d} t \sin \alpha_{n}+\varphi_{n}\right) \cdot \cos ^{2}\left(\omega_{d}(t+\tau) \sin \alpha_{n}+\varphi_{n}\right)\right\} \\
= & \frac{4}{M^{2}} \sum_{n=1}^{M} E\left[\frac{1+\cos \left(2 \omega_{d} t \sin \alpha_{n}+2 \varphi_{n}\right)}{2} \cdot \frac{1+\cos \left[2 \omega_{d}(t+\tau) \sin \alpha_{n}+2 \varphi_{n}\right]}{2}\right]
\end{aligned}
$$




$$
\begin{aligned}
=\frac{1}{M^{2}} \sum_{n=1}^{M}\left\{1+\frac{1}{2} E\left[\cos \left(2 \omega_{d} \tau \sin \alpha_{n}\right)\right]\right\} & =\frac{1}{M^{2}}\left(M+\frac{1}{2} M \cdot J_{0}\left(2 \omega_{d} \tau\right)\right) \\
& =\frac{1}{M}+\frac{1}{2 M} J_{0}\left(2 \omega_{d} \tau\right) .
\end{aligned}
$$

(2) $n=i, p=q, n \neq p$

$$
\begin{aligned}
& E\left[Z_{c}^{2}(t) Z_{c}^{2}(t+\tau)\right]=\frac{4}{M^{2}} \sum_{n=1}^{M} E\left[\cos ^{2}\left(\omega_{d} t \sin \alpha_{n}+\varphi_{n}\right)\right] \\
& \cdot \sum_{p=1}^{M} E\left[\cos ^{2}\left(\omega_{d}(t+\tau) \sin \alpha_{p}+\phi_{p}\right)\right]=\frac{4}{M^{2}} \sum_{n=1}^{M} \frac{1}{2} \cdot \sum_{p=1}^{M} \frac{1}{2}=1 .
\end{aligned}
$$

(3) $n=p, i=q, n \neq i$

$$
\begin{aligned}
E\left[Z_{c}^{2}(t) Z_{c}^{2}(t+\tau)\right]= & \frac{4}{M^{2}} \sum_{n=1}^{M} E\left[\cos \left(\omega_{d} t \sin \alpha_{n}+\varphi_{n}\right) \cdot \cos \left(\omega_{d}(t+\tau) \cos \alpha_{p}+\phi_{p}\right]\right. \\
& \cdot \sum_{i=1}^{M} E\left[\cos \left(\omega_{d} t \sin \alpha_{i}+\varphi_{i}\right) \cdot \cos \left(\omega_{d}(t+\tau) \cos \alpha_{q}+\phi_{q}\right]\right. \\
= & \frac{4}{M^{2}} \sum_{n=1}^{M}\left[\frac{1}{2} \cdot J_{0}\left(\omega_{d} \tau\right)\right] \\
& \cdot \sum_{i=1}^{M}\left[\frac{1}{2} \cdot J_{0}\left(\omega_{d} \tau\right)\right]=J_{0}^{2}\left(\omega_{d} \tau\right) .
\end{aligned}
$$

(4) $n=q, i=p, n \neq i$

Similar to the third case, one can prove

$$
E\left[Z_{c}^{2}(t) Z_{c}^{2}(t+\tau)\right]=J_{0}^{2}\left(\omega_{d} \tau\right)
$$

Therefore, from the above four kinds of circumstances, we can come to the conclusion 


$$
E\left[Z_{c}^{2}(t) Z_{c}^{2}(t+\tau)\right]=\frac{1}{M}+\frac{1}{2 M} J_{0}\left(2 \omega_{d} \tau\right)+1+2 J_{0}^{2}\left(\omega_{d} \tau\right)
$$

Using the same procedures shown above, we can obtain the following results:

$$
E\left[Z_{c}^{2}(t) Z_{s}^{2}(t+\tau)\right]=\frac{1}{M}+\frac{1}{2 M} J_{0}\left(2 \omega_{d} \tau\right)+1
$$

and

$$
E\left[Z_{s}^{2}(t) Z_{c}^{2}(t+\tau)\right]=\frac{1}{M}+\frac{1}{2 M} J_{0}\left(2 \omega_{d} \tau\right)+1
$$

Therefore, we can get

$$
R_{|z|^{2}|z|^{2}}(\tau)=4+4 J_{0}^{2}\left(\omega_{d} \tau\right)+\frac{4+2 J_{0}\left(\omega_{d} \tau\right)}{M} .
$$

\section{References}

[1] P. D. Huang, A novel structure for Rayleigh channel generation with consideration of the implementation in FPGA, IEEE Trans. on Circuits \& Systems 63(2) (2016), 216-220.

[2] S. Bose, A. Mondal and I. S. Misra, FPGA based hardware implementation of adaptive equalizer for Rayleigh fading channel, in Proc. International Conference on Microwave, Optical and Communication Engineering, India, (2015), 338-341.

[3] J. Vázquez Castillo, L. Vela-Garcia, C. A. Gutiérrez, R. Parra-Michel et al., A reconfigurable hardware architecture for the simulation of Rayleigh fading channels under arbitrary scattering conditions, AEU-International Journal of Electronics and Communications 69(1) (2015), 1-13.

[4] R. H. Clarke, A statistical theory of mobile-radio reception, Bell System Technical Journal 47(6) (1968), 957-1000.

[5] W. C. Jakes and D. C. Cox, Microwave Mobile Communications, Wiley-IEEE Press, 1994, pp. 57-66.

[6] G. E. Bottomley et al., Jakes fading model revisited, Electronics Letters 29(13) (1993), 1162-1163.

[7] Y. B. Li and Y. L. Guan, Modified Jakes model for simulating multiple uncorrelated fading waveforms, in proc. IEEE $51^{\text {st }}$ Vehicular Technology Conference Proceedings (2000), 1819-1822.

[8] Y. X. Li and X. J. Huang, The simulation of independent Rayleigh faders, IEEE Trans. on Communications 50(9) (2002), 1503-1514. 
[9] Y. R. Zheng and C. S. Xiao, Simulation models with correct statistical properties for Rayleigh fading channels, IEEE Trans. on Communications 51(6) (2003), 920-928.

[10] C. S. Patel, G. L. Stuber and T. G. Pratt, Comparative analysis of statistical models for the simulation of Rayleigh faded cellular channels, IEEE Trans. on Communications 53(6) (2005), 1017-1026.

[11] M. F. Pop and N. C. Beaulieu, Limitations of sum-of-sinusoids fading channel simulators, IEEE Trans. on Communications 49(4) (2001), 699-708.

[12] C. Xiao, Y. R. Zheng and N. C. Beaulieu, Second-order statistical properties of the WSS Jakes fading channel simulator, IEEE Trans. on Communications 50(6) (2002), 888-891.

[13] G. L. Stüber, Principles of Mobile Communication, New York: Springer Science Media, 2011, Ch. 2.

[14] Y. R. Zheng and C. Xiao, Improved models for the generation of multiple uncorrelated Rayleigh fading waveforms, IEEE Communications Letters 6(6) (2002), 256-258. 